\title{
Concepts of space, time and scale
}

Mikko Vastaranta ${ }^{1}$, Ninni Saarinen ${ }^{1,2}$, Tuomas Yrttimaa ${ }^{1}$, Timo Tokola ${ }^{1}$

${ }^{1}$ School of Forest Sciences, University of Eastern Finland, Joensuu, 80101, Finland

${ }^{2}$ Department of Forest Sciences, University of Helsinki, Helsinki, 00014, Finland; mikko.vastaranta@uef.fi, ninni.saarinen@helsinki.fi, tuomas.yrttimaa@uef.fi, timo.tokola@uef.fi

\begin{abstract}
Concepts of space, time and scale as well as their underpinning theories are crucial for understanding geospatial data. Space can be defined as a boundless, three-dimensional (3D) extent in which objects and events occur and have relative position and direction. Space has been considered to be absolute, meaning that it exists permanently and independently regardless of any matter in space. Alternatively, space can be seen as a collection of relations between objects, given by their distance and direction from one another. It has also been shown that space around the gravitational fields deviates from Euclidean space. Time, on the other hand, is considered to be part of the measuring system used to sequence events, to compare their durations and the intervals between them, as well as to quantify rates of change such as the motions of objects. However, this operational and practical definition of time does not provide arguments for whether there is really something called time, outside of counting activity that can be measured. Scale is not as controversial as space and time. The scale of a map is an important metric defining the level of detail of geoinformation that can be extracted from such a map. Only recently, scale has been started to consider as a fifth dimension.
\end{abstract}

\section{Dimensions of geospatial data}

The concepts of space, time and scale that are used in our everyday discussions without further considering the underpinning theories are first deliberated. These dimensions of geospatial data are not as trivial as they may first seem. These concepts have been the subject of many philosophical, religious, and scientific debates throughout the entire history of our societies. The simple definition for space is that it is the boundless, three-dimensional (3D) extent in which objects and events occur and have relative position and direction. In this context when dimensions of geospatial data are reviewed, the term geographical space is also often used and is considered as a synonym to land. The concept of space is considered to be fundamental for us if we want to understand the physical universe. However, there is still disagreement between 
many philosophers over whether it itself is an entity, a relationship between entities, or part of a conceptual framework. In general, entity means a thing with distinct and independent existence. Conceptual framework, on the other hand, can be defined as a network of linked concepts that together provide a comprehensive understanding of some phenomenon. There have been philosophical debates on the nature, essence, and the mode of existence of space since antiquity. For example, Platon presented his philosophical assertions on "khora" (i.e. space) and physics of Aristotle included definitions for "topos" (i.e. place). [1]

During the $17^{\text {th }}$ century, Sir Isaac Newton's view on space was absolute, conveying that it existed permanently and was independent whether there was any matter in space [2]. Gottfried Leibniz was of the different view and defined that space was a collection of relations between objects, given by their distance and direction from one another [1]. In his later works, Immanuel Kant also shared Newton's view on space.

Until around the $18^{\text {th }}$ century, and within the framework of Euclidean geometry, space was perceived by most mathematicians to be flat [1]. Then, between the nineteenth and twentieth centuries mathematicians began to examine non-Euclidean geometries, in which space was inferred to be curved, rather than flat. According to Albert Einstein's theory of general relativity, space around gravitational fields deviates from Euclidean space [3]. Furthermore, experimental tests of general relativity have confirmed that non-Euclidean space provides a better model for the shape of space [1].

Next we have time, which is considered to be part of the measuring system used to sequence events, to compare their durations, and intervals between them, as well as to quantify rates of change such as the motions of objects [1,4]. The position of events in time is changing continuously. For example, future events become present, then pass further back into the past. In the Bible, time is seen as a medium for the passage of predestined events. There is also an appointed time for everything. This can be seen as a one-way view of time. However, we should keep in mind that while we easily agree with this one-way or linear view of time, for most of humankind's existence, time has been considered cyclical and rhythmic. This cyclic view of time that has arisen from the observation of recurrences in the environment [5].

Time is one of the seven fundamental physical quantities defined in the International System of Units (SI). It is also used to define other quantities, such as velocity. An operational definition of time infers that observing a certain number of repetitions of one or another standard cyclical event (e.g. the passage of a free swinging pendulum) constitutes one standard unit such as the second [5]. This view is highly useful in the conduct of both advanced experiments and everyday affairs of life. However, this operational definition does not provide any arguments for whether there is something called time, apart from the counting activity that can be measured [1].

Two contrasting views on time divide many philosophers. The first view is that time 
is part of the fundamental structure of the universe, a dimension in which events occur in sequence. This is a realistic view of time and was supported by Sir Isaac Newton and thus, this view is sometimes referred to as Newtonian time [6]. The second and opposing view contends that time does not refer to any kind of "container" that events and objects "move through", nor to any entity that "flows", but that it is instead part of a fundamental intellectual structure together with space and number, within which humans sequence and compare events [7]. This view was supported by Gottfried Leibniz and Immanuel Kant. Many of us are familiar with Kant's famous quote regarding this view on time "Space and time are frameworks within which the mind is constrained to construct its experience of reality". It can be deduced from the this definition that time is neither an event nor a thing, and thus it itself is not measurable.

Temporal measurement has occupied the minds of scientists for a long time and was the prime motivation in the disciplines of navigation and astronomy. Cyclical (or periodic) events and cyclical motion have long served as standards for units of time. Examples include the apparent motion of the sun across the sky, the phases of the moon, the swing of a pendulum, and the beat of a heart. Actually already Aristotle has argued that time is something dependent on change as he sees it as a universal order within which all changes are related to each other [8]. Currently, the international unit of time, the second, is defined in terms of radiation emitted by cesium atoms - thus time is still defined by natural process [9]. Time is also of significant social importance and is often viewed as having economic value as captured by the popular saying "time is money", as well as personal value, due to an awareness of the limited and finite time in each day and in the human lifespan [5]. Consequently, different time scales are employed in different application domains, such as geological time, biological time, etc [1].

The dimensions of space and time have always been closely related. It is basically impossible to describe either of the two dimensions without inferring the other. For example, the standard space interval, called a standard meter or simply meter, is defined as the distance traveled by light in a vacuum during a time interval of exactly $1 / 299,792,458$ of a second [10]. Space and time represent also the space-time concept which was expressed in Einstein's special relativity and general relativity theories (Figure 1). According to these theories, the concept of time depends on the spatial reference frame of the observer and the human perception. Furthermore, the measurement by instruments such as clocks are different for observers in relative motion[5]. 


$$
\begin{aligned}
(\Delta s)^{2}=(\Delta t)^{2}-(\Delta x)^{2}-(\Delta y)^{2}-(\Delta z)^{2} \\
\text { with: } \quad \Delta x=x_{2}-x_{1} \\
\Delta y=y_{2}-y_{1} \\
\Delta z=z_{2}-z_{1} \\
\Delta t=t_{2}-t_{1}
\end{aligned}
$$

Figure 1. The spacetime interval has the same value in all reference frames. Spacetime interval is calculated between two events: $\left(\mathrm{x}_{1}, \mathrm{y}_{1}, \mathrm{z}_{1}, \mathrm{t}_{1}\right)$ and $\left(\mathrm{x}_{1}, \mathrm{y}_{1}, \mathrm{z}_{1}, \mathrm{t}_{1}\right)$ as presented above.

Advancing to the third and final dimension, namely scale. Scale has only recently started to be considered as a "dimension" [11] and is seen less controversial as space and time. Scale gives an indication of the resolution in geospatial data. In general, a larger scale means that more geospatial data would be captured, including fuzzy details that might otherwise be generalized or glossed over at smaller scales. The interpretation of scale is therefore important. For instance, by simply varying the map scale alone, the estimated distance between two points would vary which is not desired. For many years, the dimension of scale was not explicitly integrated into data modeling. Therefore, scale was assumed to be uniform within a spatio-temporal context.

The presented three rather different views of space affect how geospatial data is presented and interpreted. Relativistic view of space, championed by Leibniz, is applied in most of the geographical research [12], in studies related to spatial processes, for example. Space is also defined by the spatial elements and processes under consideration, such as migration and commuting patterns, watersheds, dispersion of pollutants, and even the diffusion of ideas and information. This approach may result in space being defined in non-Euclidean terms. In some cases, even distance may be relative as two areas separated by a barrier may be close in absolute space and very distant in relative space when time, rates, and interactions are considered.

Absolute space, championed by Newton and latterly Kant, is used in remote sensing, inventory, planning, and cartography. Space may exist for its own sake independent of matter. In other words, the job of geoinformation science should mainly consist of filling the 'container' with information. Absolute space and scale involve primarily an Euclidean viewpoint usually based on a defined grid system. When analysing spatial processes we should also consider whether we have a linear or cyclical view of time. As presented earlier, space and time are closely related due to how meter is defined, for example. Moreover, scale is the third important dimension for usage and understanding of geospatial data. To conclude, 3D space captures the geometrical characteristics of geospatial data, 4D integrates the temporal representation, while 5D also provides the scale definition. 
Author Contributions: Writing — original draft preparation, M.V.; writing — review and editing, all the authors.

\section{References}

1. Awange, J.L.; Kyalo Kiema, J.B. Geodata and Geoinformatics. Environmental Geoinformatics 2013, 17-27.

2. French, A.J.; Ebison, M.G. Introduction to CLASSICAL MECHANICS; Springer Science \& Business Media, 2012; ISBN 9789400941199.

3. Carnap, R. An Introduction to the Philosophy of Science; Courier Corporation, 2012; ISBN 9780486140865.

4. Internet Encyclopedia of Philosophy I An encyclopedia of philosophy articles written by professional philosophers Available online: https://www.iep.utm.edu/ (accessed on Mar 18, 2020).

5. Contributors to Wikimedia projects Time - Wikipedia Available online: https://en.wikipedia.org/wiki/Time (accessed on Apr 9, 2020).

6. Internet Encyclopedia of Philosophy I An encyclopedia of philosophy articles written by professional philosophers Available online: https://www.iep.utm.edu/ (accessed on Mar 18, 2020).

7. Internet Encyclopedia of Philosophy I An encyclopedia of philosophy articles written by professional philosophers Available online: https://www.iep.utm.edu/ (accessed on Mar 18, 2020).

8. Coope, U. Time as a Measure of Change. Time for Aristotle 2005, 99-110.

9. Contributors to Wikimedia projects Second - Wikipedia Available online: https://en.wikipedia.org/wiki/Second (accessed on Apr 9, 2020).

10. Contributors to Wikimedia projects Metre - Wikipedia Available online: https://en.wikipedia.org/wiki/Metre (accessed on Apr 9, 2020).

11. van Oosterom, P.; Stoter, J. 5D Data Modelling: Full Integration of 2D/3D Space, Time and Scale Dimensions. Geographic Information Science 2010, 310-324.

12. Meentemeyer, V. Geographical perspectives of space, time, and scale. Landscape Ecology 1989, 3, 163-173. 